\title{
Adaptación de material de rehabilitación para su uso en otras culturas
}

\author{
Nuria Menéndez Álvarez ${ }^{a}$, Estíbaliz Jiménez Arberas ${ }^{\text {y }}$ María Luisa Ruiz ${ }^{\mathrm{c}}$ \\ ${ }^{a}$ Doctoranda en psicología en la Universidad de Salamanca. Terapeuta Ocupacional (Facultad Padre Ossó) \\ nuria_proaza@hotmail.com, ${ }^{b}$ Coordinadora del Grado en Terapia Ocupacional de la Facultad Padre Ossó , ${ }^{\text {CProfesora }}$ \\ del Grado en Terapia Ocupacional de la Facultad Padre Ossó
}

\begin{abstract}
Through this project, the students of the subject of Autonomy and functional independence in social exclusion of the Degree in Occupational Therapy of the Padre Ossó Faculty, have elaborated several sequences of pictograms respecting iconicity to enable their use in other cultures, specifically in the camps. of Sahrawi refugees from Tindouf (Algeria). The elaboration of the pictograms has brought the students to a completely different reality from the one they know, thus instilling values of sensitivity and solidarity among university youth. Likewise, through this project the development of relevant cross-cutting themes such as interculturality, cooperation and development education has been promoted.
\end{abstract}

Keywords: Pictograms, cooperation, interculturality, iconicity

\section{Resumen}

A través del presente proyecto el alumnado de la asignatura de Autonomía e independencia funcional en exclusión social del Grado en Terapia Ocupacional de la Facultad Padre Ossó, ha elaborado varias secuencias de pictogramas respetando la iconicidad para posibilitar su uso en otras culturas, concretamente en los campamentos de refugiados saharauis de Tindouf (Argelia). La elaboración de los pictogramas, ha acercado a los estudiantes a una realidad completamente diferente a la que conocen, inculcando de esta forma, valores de sensibilidad y solidaridad entre la juventud universitaria. De igual forma, a través del presente proyecto se ha fomentado el desarrollo de temáticas transversales relevantes como son la interculturalidad, la cooperación y la educación para el desarrollo.

Palabras clave: Pictogramas, cooperación, interculturalidad, iconicidad

\section{Introducción}

Los campamentos de refugiados saharauis están localizados en la Hamada argelina, uno de los territorios más inhóspitos de la tierra donde llegan a alcanzarse temperaturas de más de 50 grados. En este territorio los sahrauis llevan asentados en situación de refugio desde hace más de cuarenta años. Dentro de su organización política y social han intentado paliar las necesidades de toda la población con la ayuda humanitaria procedente de varios países del mundo. En este sentido, de cara a prestar servicio a la población con discapacidad se ha creado en cada wilaya (ciudad) un centro de educación especial.

Estos centros son coordinados por personal saharaui voluntario, sin ningún tipo de formación en discapacidad. A estos centros acceden niños que son capaces de realizar la marcha de manera 
independiente, siendo las patologías con mayor prevalencia parálisis cerebral, autismo y síndrome de Down. Estas patologías, se caracterizan por presentar dificultades en la comunicación y diferentes transtornos del lenguaje.

Es conocido en nuestra sociedad, el uso de pictogramas como herramienta educativa y rehabilitadora que facilite la comunicación. En el caso de la población saharaui, tal y como se ha mencionado anteriormente, son totalmente dependientes de la ayuda humanitaria. En este sentido, los pictogramas de los que se disponía en los diferentes centros de educación especial estaban donados por entidades occidentales, que mostraban ocupaciones occidentales. Dado que los pictogramas deben respetar criterios de iconicidad, es decir representar lo más fielmente posible a la realidad la imagen u ocupación que representa, los pictogramas disponibles en los campamentos no cumplian este criterio $\mathrm{y}$, por tanto, no podían ser utilizados.

Es importante comprender la importancia de la cultura, entorno y contexto cuando se trabaja con poblaciones diferentes a la propia. Por ejemplo, actividades como la alimentación o la higiene personal se realizan de manera diferentes en las distintas partes del mundo. Por ello, el material con el que se trabajen dichas actividades debe ser adaptado al colectivo con el que se trabaje.

En este sentido, surgió el proyectode innovación docente "Adaptación de material de rehabilitación para su uso en otras culturas" que se llevó a cabo durante el segundo semestre del curso 2019/2020 con el alumnado de la asignatura Autonomía e independencia funcional en exclusión social del grado de Terapia Ocupacional de la Facultad Padre Ossó, centro adscrito a la Universidad de Oviedo. (PIN-A-063).

Se ha planteado el proyecto al alumnado de Terapia Ocupacional dado que, la Terapia Ocupacional es la profesión sociosanitaria que se encarga de maximizar la independencia de los diferentes individuos en las actividades de la vida diaria. Es decir, el terapeuta ocupacional dispone de los conocimientos necesarios para intervenir en las diferentes ocupaciones que realiza la persona independientemente del entorno y contexto que le rodee. Esto hace que la Terapia Ocupacional tenga un amplio campo de actuación en Cooperación al desarrollo.

A través del presente proyecto los alumnos elaboraron varias secuencias de pictogramas relacionados con diferentes actividades básicas de la vida diaria. Dichos pictogramas debían respetar en todo momento la iconicidad para permitir su uso en otras culturas, concretamente en los campamentos de refugiados sahrauis localizados en Tindouf (Argelia) donde fueron enviados en febrero de 2020.

La elaboración de los pictogramas pretendía acercar a los estudiantes a una realidad completamente diferente a la que conocen, inculcando, de esta forma, valores de sensibilidad y solidaridad entre la comunidad universitaria.. De igual forma, a través del presente proyecto, se ha fomentado el desarrollo de temáticas transversales relevantes como son la interculturalidad, la cooperación y la educación para el desarrollo. Desde mayo de 2019, la Universidad de Oviedo mantiene un convenio de colaboración con la Universidad de Tifariti y la Delegación Saharaui en Asturias, en el que se comprometen, entre otras cosas, al desarrollo e intercambio de materiales para la investigación y la enseñanza, objetivos a los que se contribuye con el presente proyecto.

\section{Objetivos}

Los objetivos del proyecto “Adaptación de material de rehabilitación para su uso en otras culturas son”:

- Reflexionar sobre la interculturalidad en Terapia Ocupacional 
- Educar al alumnado en valores de igualdad y solidaridad, así como fomentar la apreciación de la diversidad y multiculturalidad.

- Comprender y reconocer la interacción entre conceptos propios de la disciplina de Terapia Ocupacional como ocupación,entorno y contexto.

- Reconocer la influencia de las diferencias individuales, así como de las costumbres sobre ocupación y participación.

- Analizar y comprender la figura del terapeuta ocupacional en ámbitos de apartheid ocupacional y justicia ocupacional

- Conocer las características básicas de los pictogramas

- Trabajar y reflexionar sobre la iconicidad en diferentes entornos y contextos

\section{Desarrollo de la innovación}

Tal y como se ha indicado anteriormente, el proyecto se desarrolló en la facultad Padre Ossó durante el segundo semestre del curso 2019/2020 bajo el marco de la asignatura Autonomía e independica funcional en exclusión social.

La exclusión social es un fenómeno dinámico y multicausal en continuo cambio debido al proceso de transformación social, dentro de la asignatura de Autonomía e Independencia funcional en exclusión social, se abordan los diferentes colectivos en riesgo de exclusión social susceptibles de intervención desde Terapia Ocupacional. Uno de estos colectivos, son personas refugiadas, como es el caso de la población saharaui. Por lo tanto, a través del proyecto los alumnos han reforzado los conocimientos adquiridos durante las clases expositivas, así como conocido nuevos ámbitos de actuación. Como ya se ha indicado anteriormente, el terapeuta ocupacional cumple el perfil idóneo para trabajar en el ámbito de la cooperación al desarrollo (Sánchez y Moro, 2015), sin embargo, aún son pocos los profesionales que se dedican a este ámbito.

Se ha decidio la realización de pictogramas como material de rehabilitación ya que, el Ministerio de Asuntos Sociales de la República Árabe Saharaui Democrática (RASD) llevaba solicitando este material desde hace varios años, al verse limitado en el trabajo a realizar en los centros de educación especial (tanto a nivel comunicativo, como de trabajo de rutinas y actividades de la vida diaria) por la falta de material. Por otro lado, se decide que sean alumnos de Terapia Ocupacional quien lleve a cabo el proyecto dada la importancia que la ocupación tiene en la disciplina y lo variable que resulta la ocupación en función de la cultura, el entorno y el contexto.

A través de la elaboración de los pictogramas, se ha acercado a los estudiantes a una realidad completamente diferente a la que conocen, inculcando de esta forma, valores de sensibilidad y solidaridad entre la juventud universitaria. De igual forma, a través del presente proyecto se ha fomentado el desarrollo de temáticas transversales relevantes como son la interculturalidad, la cooperación y la educación para el desarrollo. La Asociación Asturiana de Solidaridad con el Pueblo Saharaui lleva desde el año 2017 realizando intervenciones de Terapia Ocupacional en los campamentos de refugiados, concretamente en las escuelas de educación especial en las que se han entrego los pictogramas elaborados.

El proyecto se ha desarrollado a través de una metodología teórico práctica. Al inicio del proyecto, durante la segunda mitad de enero y primera quincena de febrero, la persona encargada del ámbito de la 
discapacidad en la Asociación Asturiana de Solidaridad con el Pueblo Saharaui llevo a cabo doce horas de clases expositivas con el objetivo de explicar el proyecto que la asociación lleva a cabo en los campamentos de refugiados saharauis y acercar a los alumnos a una nueva realidad de intervención. Una vez finalizadas las clases expostivas, se dividió a la clase en cuatro grupos.

Durante las clases prácticas, coincidiendo éstas con las prácticas de aula de la asignatura, cada grupo se dedicó a la elaboración de una secuencia completa de pictogramas de las siguientes actividades: Vestirse, orinar defecar y ducharse. Se decidió que fuesen estas y no otras las secuencias de actividades a realizar porque todas ellas son actividades básicas de la vida diaria. Es decir, todas ellas son tareas que la persona realiza de manera diaria y por tanto, tendrán un mayor impacto en su vida a nivel de independencia y autonomía, que otro tipo de actividades menos frecuentes.
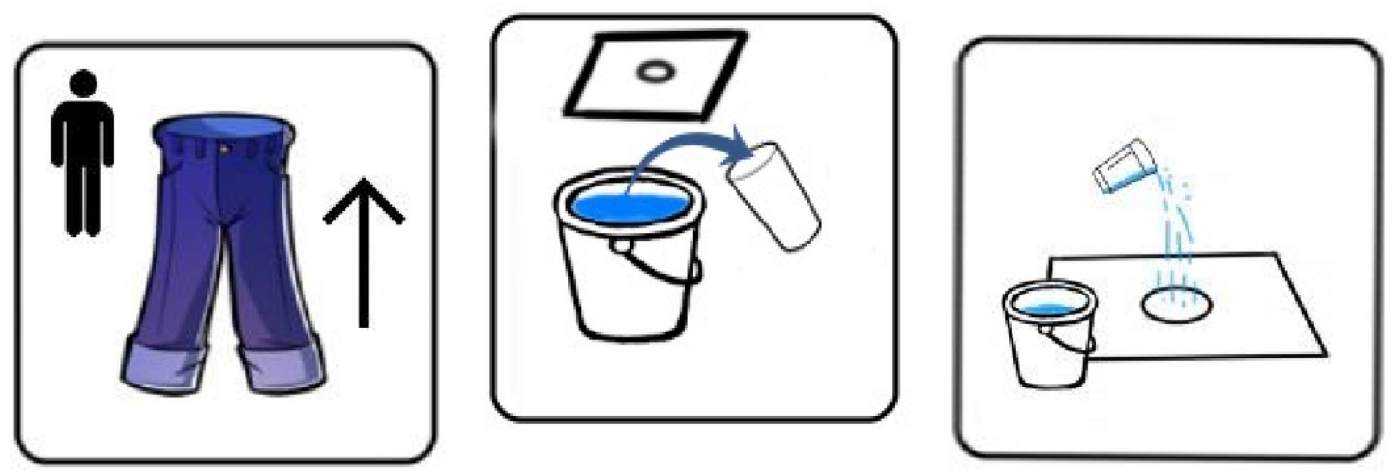

Ilustración 1 Ejemplos de pictogramas elaborados

Para facilitar la realización del trabajo se les proporcionó a los alumnos material fotográfico del entorno saharaui. Respecto a la elaboración de los pictogramas, se permitió que cada grupo eligiese el programa informático o manual con el que más cómodos se sintiesen siempre y cuando, la impresión pudiese ajustarse al tamaño adecuado.

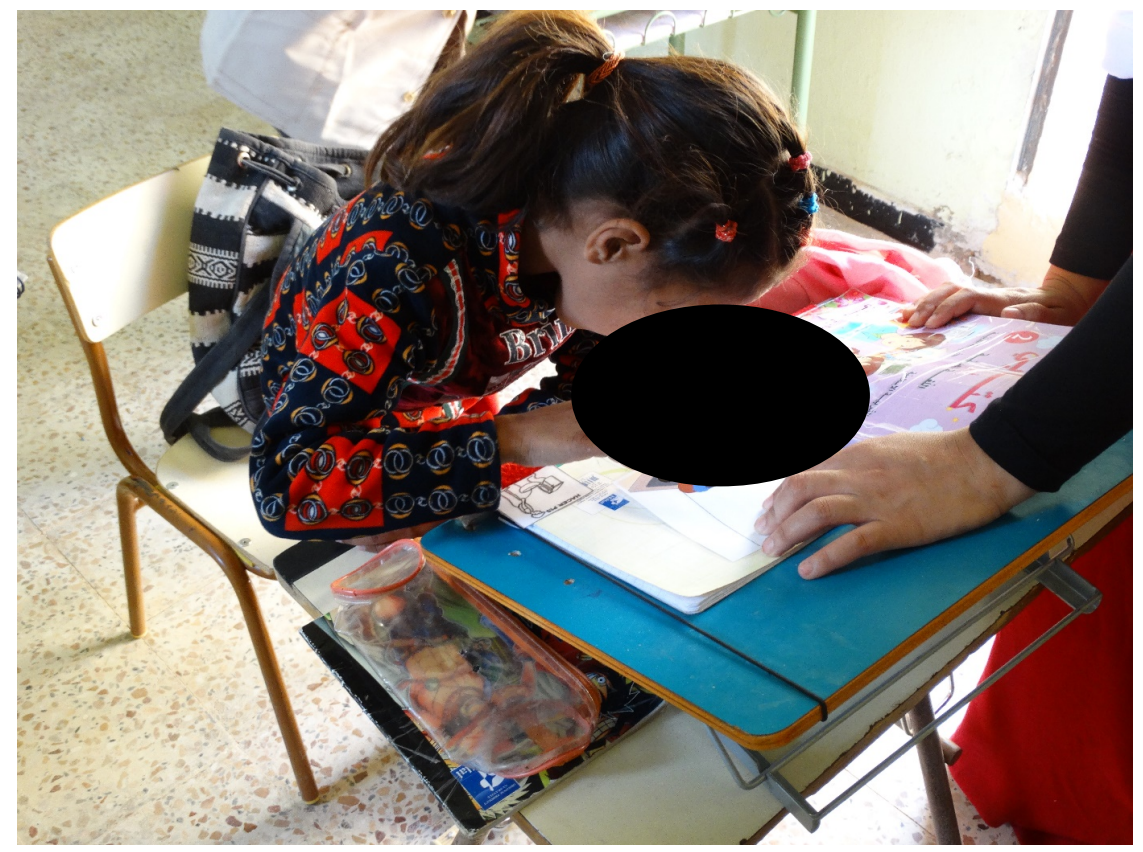

Ilustración 2 Alumna saharaui utilizando uno de los pictogramas elaborados por el alumnado de la Facultad Padre Ossó 
Una vez finalizados los pictogramas, fueron entregados a la responsable de discapacidad en la Asoaciación Asturiana de Solidaridad con el Pueblo Saharaui, que a su vez los ha entregado en el centro de educación especial de la wilaya de Auserd del 22 al 29 de febrero de 2020, coincidiendo con el viaje en el que la asociación ha realizado su proyecto de Terapia Ocupacional.

Durante este viaje, se ha comprobado la iconicidad de los pictogramas realizados por los alumnos y se ha entregado a los mismos feedback a través de material fotográfico y audiovisual por parte de los niños saharauis beneficiados del material creado como de los docentes que se han visto beneficiados de su utilidad en el aula.

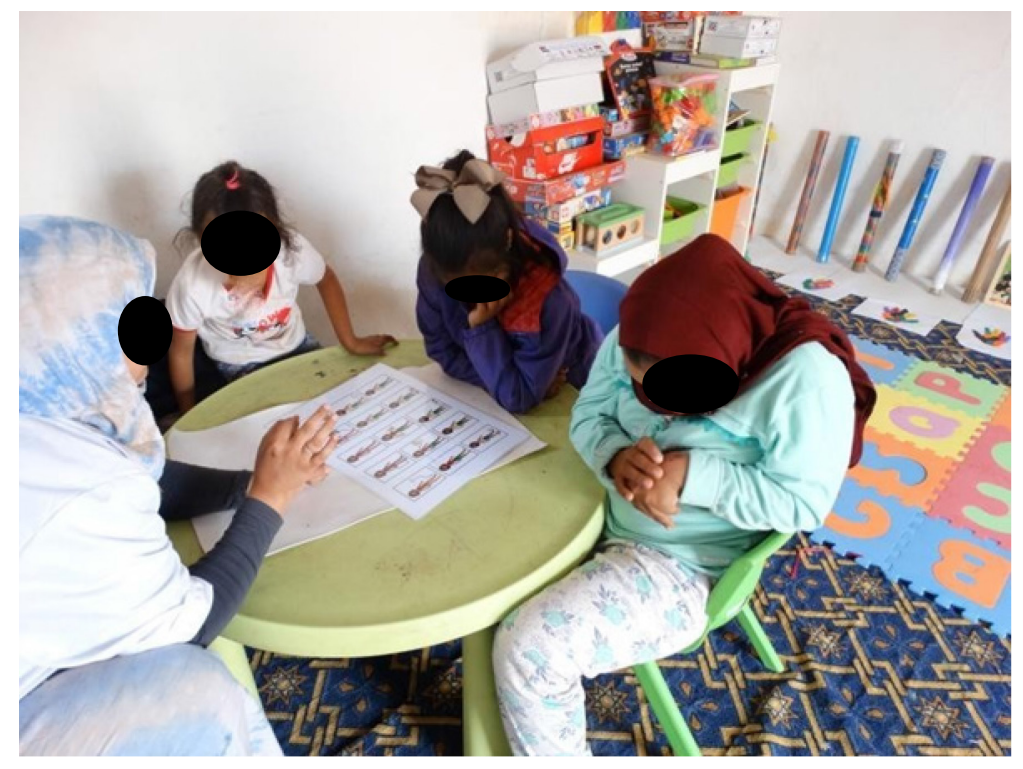

Ilustración 3 Entrega de los pictogramas en la escuela de educación especial de Auserd

El proyecto presentado ha resultado una práctica innovadora, que ha generado gran interés y motivación entre el alumnado. Además, se debe destacar, que la realización de este proyecto no sólo favorece al alumando de la asignatura de Autonomía e independencia funcional en exclusión social. A su vez, el alumnado y personal docente de los centros de educación especial de los campamentos de refugiados saharauis también se ve enormemente beneficiado. El pictograma representa de manera visual actividades cotidianas y facilita la comunicación (Cáceres Acosta, 2017). La principal característica del pictograma es la iconicidad, entendiéndose ésta por el grado en el que un símbolo se parece a lo que representa (Bertola López, 2017). Por ello, el pictograma tiene en cuenta los significados culturales y sociales (García y Cruz, 2015). En la actualidad, los pictogramas disponibles en las escuelas de educación especial de los campamentos de refugiados saharauis han sido exportados de la cultura occidental lo que genera que las actividades representadas no son significativas para la población a la que se dirigen y, por lo tanto, no cumplen los objetivos pedagógicos y terapéuticos que se busca en su utilización. A través de la realización de los pictogramas adaptados a su cultura y contexto el alumnado saharaui podrá identificar y comprender los acontecimientos importantes de su entorno, comprender el mundo que le rodea, entender y expresar sus emociones y facilitar una forma de comunicación ( Regis y callejón, 2015) lo que facilitará la participación del alumnado saharaui con necesidades especiales en las actividades de la vida diaria. 


\section{Resultados}

Los resultados del presente proyecto han resultado positivos tanto para el alumnado como para el personal docente y colaborador participante.

Han participado en el proyecto un total de 26 alumnos de la Facultad Padre Ossó y 2 profesoras del mismo centro. De manera indirecta, se ha beneficiado del proyecto un total de 20 alumnos del centro de educación especial de Auserd y 4 trabajadoras del centro.

Para la obtención de los resultados se ha evaluado el documento presentado (secuencia de pictogramas), la participación en el aula y la satisfacción del alumno.

Respecto al alumnado, el grado de interés sobre las actividades propuestas durante la realización del proyecto ha sido de 9,13 sobre 10 . Por otro lado, en relación a la utilidad del proyecto con su futuro profesional solamente un alumno del total de los participantes considera que los temas tratados no influirán en su futuro profesional.

Académicamente, los resultados obtenidos en la elaboración de los pictogramas han sido buenos, siendo la calificación más baja de 7,la más alta de 8,3y resultando la media total de 7,74. Para la calificación de estos resultados se ha tenido en cuenta un $70 \%$ el trabajo presentado y un $15 \%$ la participación en el aula. Dentro del $70 \%$ de valoración del documento se ha tenido en cuenta que cumpliese los siguientes criterios: Iconicidad (3 puntos), adaptación al contexto ( 3 puntos), usabilidad (3 puntos) y presentación (1 punto).

Cabe destacar, que el 100\% del alumando volvería a participar en un proyecto similar. Además, destacan positivamente el hecho de que el trabajo realizado tuviese una utilidad real, mostrando en la encuesta de satisfacción comentarios como "Considero que este tipo de proyectos son retos para nosotros, nos aportan experiencias y conocimientos, además de que nos ayudan a salir de nuestra zona de confort".

Por otro lado, tanto el personal docente como el personal colaborador califican de positivo el desarrollo y resultado final del proyecto "Adaptación de material de rehabilitación para su uso en otras culturas".

\section{Conclusiones}

Proyectos como el descrito en el presente documento permiten al alumnado universitario reflexionar sobre las competencias de su futura profesión en ámbitos poco frecuentes en la disciplina de Terapia Ocupacional. Así mismo, refuerza entre los estudiantes valores de solidaridad y cooperación tan olvidados y necesarios en la sociedad actual. Por otro lado, el hecho de que la práctica tenga un fin y una utilidad motiva al alumando en la ejecución de la misma, al verse empoderados por la utilidad real que el trabajo realizado tiene para otras poblaciones.

Han sido varios los alumnos que, una vez finalizada la práctica, se han mostrado interesados en seguir colaborando en diferentes acciones de solidaridad en las escuelas de educación especial saharaui. Además, se ha solicitado la ampliación del proyecto para continuar realizandolo durante el curso 2020/21.

Por otro lado, también se está trabajando en la continuadad de la actividad a través del proyecto "Tendiendo Puentes Construyendo Capacidades" que desarrolla la Facultad Padre Ossó y Medicusmundi en el Centro de atención y control nutricional materno infantil de Kalana (Mali), donde sería necesario adaptar los pictogramas a un nuevo entorno y cultura. 
Además, en el mes de octubre, se llevará a cabo un taller titulado "cooperación al desarrollo y actividades de la vida diaria: Terapia Ocupacional en los campamentos de refugiados saharauis"en el que se expondrá de forma práctica el proyecto dentro de las jornadas "Genero y discapacidad" en las que se espera una inscripción superior a los 100 participantes.

\section{Agradecimientos}

Agradecer a los alumnos de $2^{\circ}$ del Grado de Terapia Ocupacional de la Facultad Padre Ossó su disposición, implicación y buen hacer durante todo el desarrollo del proyecto.

\section{Referencias bibliográficas}

Bertola López, E. (2017) Análisis empírico de las características formales de los símbolos pictográficos ARASAAC. Tesis Doctoral. Universidad de Murcia.

Cáceres Acosta, O.(2017) El uso del pictograma en el proceso de enseñanza aprendizaje del niño con autismo. Tesis doctoral. Universidad de las Palmas de Gran Canaria.

García, J. M. P., \& de la Cruz, L. L. (2015). La lectura fácil: una apuesta de valor para las organizaciones. Revista Española de Discapacidad (REDIS), 3(1), 187-92.

Regis, P; Callejón, M. (2015) Del pictograma a la imagen: Herramientas de comunicación y lenguaje en personas con síndorme Asperger a través de recursos visuales para la inclusión social. Arteterapia (10), 329-341

Sánchez, L \& Moro, L. (2015). Intervención de Terapia Ocupacional para personas con discapacidad en un campo de refugiados palestinos en la franja de gaza, Revista gallega de Terapia Ocupacional, 12(21). 